\title{
The 2011 outburst of the recurrent nova T Pyxidis. Evidence for a face-on bipolar ejection
}

\author{
O. Chesneau ${ }^{1}$, A. Meilland ${ }^{1}$, D. P. K. Banerjee ${ }^{2}$, J.-B. Le Bouquin ${ }^{3}$, H. McAlister ${ }^{4,5}$, F. Millour ${ }^{1}$, S. T. Ridgway ${ }^{6}$, \\ A. Spang ${ }^{1}$, T. ten Brummelaar ${ }^{5}$, M. Wittkowski ${ }^{7}$, N. M. Ashok ${ }^{2}$, M. Benisty ${ }^{8}$, J.-P. Berger ${ }^{9}$, T. Boyajian ${ }^{4}$, \\ Ch. Farrington ${ }^{5}$, P. J. Goldfinger ${ }^{5}$, A. Merand ${ }^{9}$, N. Nardetto ${ }^{1}$, R. Petrov ${ }^{1}$, Th. Rivinius ${ }^{9}$, G. Schaefer ${ }^{4}$, \\ Y. Touhami ${ }^{4}$, and G. Zins ${ }^{3, \star}$
}

\author{
${ }^{1}$ UMR 6525 Fizeau, Univ. Nice Sophia Antipolis, CNRS, Obs. de la Côte d'Azur, Bvd de l'Obs., BP 4229, 06304 Nice Cedex 4 , \\ France \\ e-mail: Olivier.Chesneau@oca.eu \\ 2 Physical Research Laboratory, Navrangpura, Ahmedabad, Gujarat, India \\ ${ }^{3}$ UJF-Grenoble 1/CNRS-INSU, Institut de Planétologie et d'Astrophysique de Grenoble (IPAG), UMR 5274, Grenoble, France \\ ${ }^{4}$ Georgia State University, PO Box 3969, Atlanta GA 30302-3969, USA \\ 5 CHARA Array, Mount Wilson Observatory, 91023 Mount Wilson CA, USA \\ ${ }^{6}$ National Optical Astronomy Observatories, 950 North Cherry Avenue, Tucson, AZ, 85719, USA \\ 7 European Southern Observatory, Karl-Schwarzschild-Strasse 2, 85748 Garching bei München, Germany \\ 8 Max Planck Institut für Astronomie, Königstuhl 17, 69117 Heidelberg, Germany \\ 9 European Southern Observatory, Casilla 19001, Santiago 19, Chile
}

Received 29 July 2011 / Accepted 28 September 2011

\section{ABSTRACT}

\begin{abstract}
Aims. T Pyx is the first recurrent nova ever historically studied. It was seen in outburst six times between 1890 and 1966 and then not for 45 years. We report on near-IR interferometric observations of the recent outburst of 2011.

Methods. We obtained near-IR observations of TPyx at dates ranging from $t=2.37 \mathrm{~d}$ to $t=48.2 \mathrm{~d}$ after the outburst, with the CLASSIC recombiner located at the CHARA array and with the PIONIER and AMBER recombiners located at the VLTI array. These data are supplemented with near-IR photometry and spectra obtained at Mount Abu, India. We compare expansion of the $H$ and $K$ band continua and the $\operatorname{Br} \gamma$ emission line, and infer information on the kinematics and morphology of the early ejecta.

Results. Slow expansion velocities were measured $\left(\leq 300 \mathrm{~km} \mathrm{~s}^{-1}\right)$ before $t=20 \mathrm{~d}$. From $t=28 \mathrm{~d}$ on, the AMBER and PIONIER continuum visibilities ( $K$ and $H$ band, respectively) are best simulated with a two-component model consisting of an unresolved source plus an extended source whose expansion velocity onto the sky plane is lower than $\sim 700 \mathrm{~km} \mathrm{~s}^{-1}$. The expansion of the Br lineforming region, as inferred at $t=28 \mathrm{~d}$ and $t=35 \mathrm{~d}$, is slightly larger, implying velocities in the range $500-800 \mathrm{~km} \mathrm{~s}^{-1}$, which is still strikingly lower than the velocities of $1300-1600 \mathrm{~km} \mathrm{~s}^{-1}$ inferred from the Doppler width of the line. Moreover, a remarkable pattern was observed in the $\mathrm{Br} \gamma$ differential phases. A semi-quantitative model using a bipolar flow with a contrast of 2 between the pole and equator velocities, an inclination of $i=15^{\circ}$, and a position angle $\mathrm{PA}=110^{\circ}$ provides a good match to the AMBER observables. At $t=48 \mathrm{~d}$, a PIONIER dataset confirms the two-component nature of the $H$ band emission, consisting of an unresolved stellar source and an extended region whose appearance is circular and symmetric within error bars.

Conclusions. These observations are most simply interpreted within the frame of a bipolar model, oriented nearly face-on. This finding has profound implications for interpreting past, current, and future observations of the expanding nebula.
\end{abstract}

Key words. circumstellar matter - techniques: high angular resolution - novae, cataclysmic variables - stars: individual: T Pyx

\section{Introduction}

A classical nova eruption results from a thermonuclear runaway on the surface of a white dwarf that is accreting material from a companion star in a close binary system. T Pyxidis (T Pyx) is a unique recurrent nova that was in outburst six times between 1890 and 1966 (intervals of $\sim 20 \mathrm{yr}$ ). T Pyx was discovered in outburst at a visual magnitude of 13.0 on 2011 April 14.29 UT $(\mathrm{JD}=2455665.79)$, which we take as $t_{0}=0$ (Waagan et al. 2011). This is the first outburst of T Pyx since December 7, 1966, nearly 45 years before.

* Based on observations made with CHARA at Mount Wilson observatory and the VLTI at Paranal Observatory under program 287.D5012, 287.D-5023, 087.C-0702.
The evolution of the nova is relatively slow, thereby providing time and scope for organizing joint observations with optical interferometry arrays such as CHARA and the VLTI. T Pyx is surrounded by an interesting nebula in expansion that has been investigated by the HST for more than ten years (Schaefer et al. 2010, and references therein). The knots are expanding in the plane of the sky with velocities ranging from roughly 500 to $715 \mathrm{~km} \mathrm{~s}^{-1}$. In contrast, the velocities inferred from Doppler widths of the ejecta of recent outbursts were observed to be much faster at about $1500 \mathrm{~km} \mathrm{~s}^{-1}$. Although T Pyx is a well-observed system, it still has many mysteries. Why did the ejecta expand so slowly in the plane of the sky? An important spectroscopic study of the binary system by Uthas et al. (2010) provides evidence of a low inclination for the system orbit $\left(i=10 \pm 2^{\circ}\right)$, a particularly 
Table 1. Journal of interferometric observations.

\begin{tabular}{lccccccc}
\hline \hline Date & $\begin{array}{c}\text { MJD } \\
2450000.5+\end{array}$ & $\begin{array}{c}t-t_{0} \\
\text { phase }\end{array}$ & Instrument & Base & \multicolumn{2}{c}{$\begin{array}{c}\text { Projected baselines } \\
\text { length [m] }\end{array}$} & PA ${ }^{\circ}$ ] \\
\hline $2011 / 04 / 17$ & 5668.16 & 2.92 & CLASSIC & W1-W2 & 107.4 & -87 & HD 78752, HD 79290 \\
$2011 / 04 / 23$ & 5674.04 & 8.81 & AMBER & K0-A1-I1 & $45.4 / 100.3 / 120.2$ & $-159.0 / 96.2 /-105.0$ & HD 73947 \\
$2011 / 04 / 26$ & 5678.06 & 12.81 & PIONIER & A1-G1-I1-K0 & $74 / 94 / 42 / 113 / 82 / 44$ & $-52 /-74 /-127 / 81 / 39 / 23$ & HD 78739 \\
$2011 / 04 / 28$ & 5679.18 & 13.93 & CLASSIC & W1-E2 & $202.1 / 213.3$ & $-79.4 / 82.8$ & HD 78752, HD 79290 \\
$2011 / 05 / 12$ & 5694.00 & 28.76 & AMBER & UT1-3-4 & $59.5 / 95.6 / 117.7$ & $127.1 / 43.1 / 73.3$ & HD 73947 \\
$2011 / 05 / 20$ & 5701.02 & 35.77 & AMBER & UT1-3-4 & $56.2 / 89.9 / 105.6$ & $136.9 /-133 / 78.6$ & HD 73947, HD 87303 \\
$2011 / 06 / 01$ & 5713.99 & 48.74 & PIONIER & D0-G1-H0-I1 & $68 / 47 / 63 / 67 / 37 / 40$ & $171 / 96 / 132 / 25 / 57 / 180$ & HD 78739 \\
\hline
\end{tabular}

Notes. ${ }^{(a)}$ Calibrator angular diameters from SearchCal@JMMC (Bonneau et al. 2006): HD 78752 (G0V, $0.22 \pm 0.02$ mas), HD 79290 (A0V, $0.13 \pm 0.01$ mas), HD 73947 (K2III, $0.86 \pm 0.02$ mas), HD 87303 (K2III, $0.90 \pm 0.07$ mas), HD 78739 (K0III, $0.32 \pm 0.02$ mas).

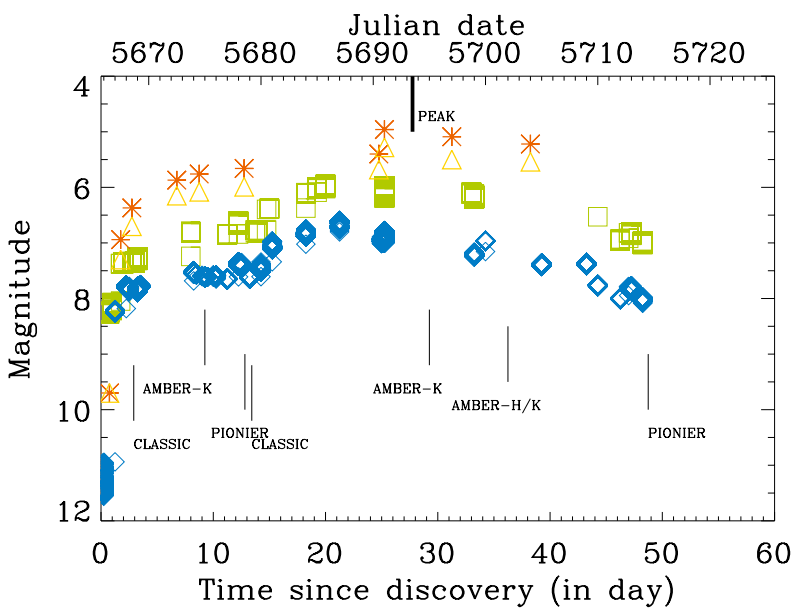

Fig. 1. Light curve of T Pyx with the dates of the optical interferometry observations. Blue diamonds indicate a subset of AAVSO data in $V$, and green squares in $I$. Orange triangles and red stars indicate $H$ and $K$ band photometry from Mt Abu (India).

important constraint for interpreting of interferometric data, as it appears that the ejecta emitted around these outbursting sources are rarely spherical.

This letter presents optical interferometry measurements obtained from different facilities that provide important information when included within a common frame of interpretation. The observations are presented in Sect. 2. In Sect. 3 we analyze the continuum measurements by means of simple geometrical model and the differential observables through the $\mathrm{Br} \gamma$ line using a simple model, and then discuss the results in Sect. 4.

\section{Observations}

Near-infrared JHK photometric and spectroscopic observations were obtained on a regular basis from the $1.2 \mathrm{~m}$ telescope at the Mt. Abu Observatory, India. These measurements helped to prepare the interferometric observations and to evaluate the relative contribution of the various continuum and line components (Fig. 1). Initial observations are reported in Banerjee \& Ashok (2011), and a fuller study is in preparation.

Prompt broad-band interferometric observations were secured with CLASSIC, a two-telescope high-sensitivity system located at CHARA on Mt. Wilson (ten Brummelaar et al. 2005). Despite the faintness and low declination of the source, observations in the $K$-band were obtained at $t=2.92 \mathrm{~d}(K \approx 6.4$, from Mt Abu observations) and $t=13.93 \mathrm{~d}(K \approx 5.7)$. The $\log$ of the observations is presented in Table 1 and the data in Fig. 2.

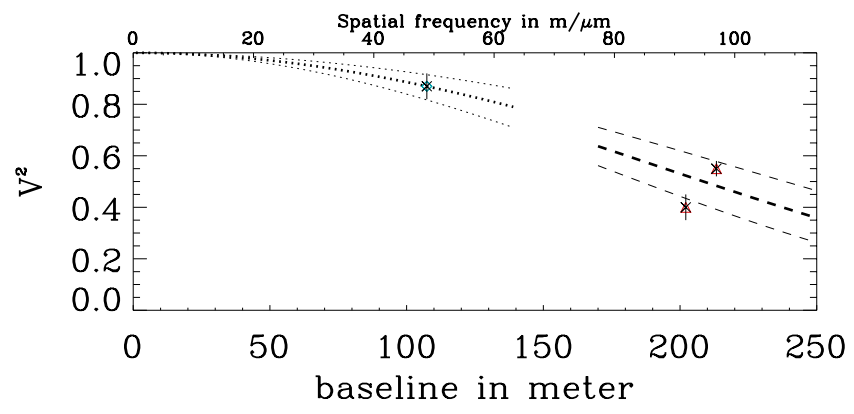

Fig. 2. $K$-band interferometric visibilities obtained with CLASSIC at $t=2.92$ (green diamond) and $t=13.93$ (red triangles). The thick dotted and dashed lines indicate are the UD curves corresponding to Table 2.

Several interferometric observations at medium spectral resolution $(R=1500)$ across the $\mathrm{Br}-\gamma$ line were obtained with AMBER, a three-telescope combiner located at the VLTI (Petrov et al. 2007). The first observations were performed with the $1.8 \mathrm{~m}$ Auxiliary Telescopes (ATs) at $t=8.81 \mathrm{~d}$, when the source was below the interferometric sensitivity limit of AMBER ( $K \approx 5.7$ ), but a useful spectrum was obtained. The second and third measurements, obtained with the $8.2 \mathrm{~m}$ Unit Telescopes (UTs) at $t=28.76(K \approx 4.9)$ and $t=35.77 \mathrm{~d}(K \approx 5)$, produced good quality dispersed visibilities, closure and differential phases (see Fig. 5). Unfortunately, the calibrator measurement for the last date is of poor quality, preventing any reliable calibration of the absolute visibility.

Imaging broad-band interferometric observations were obtained at $t=12.81 \mathrm{~d}(H \approx 6)$ with the PIONIER visitor instrument (Berger et al. 2010; Le Bouquin et al. 2011). These observations provided the simultaneous measurement of six absolutely calibrated visibilities and four closure phases in the $H$-band, therefore allowing the study of the spatial morphology of the near-infrared emission. A critical second observation was obtained at $t=48.74 \mathrm{~d}(H \approx 6)$, again with the ATs (Fig. 3).

\section{Analysis}

The absolute visibility measurements were fitted with simple geometrical models using the LITpro software (Tallon-Bosc et al. 2008, JMMC). The results are shown in Table 2. A simple uniform disk (UD) model, i.e. a circular disk of uniform brightness in the plane of the sky, was fitted to the measurements for the early observations. For later observations, a twocomponent model, consisting of an unresolved component, and a co-centered uniform disk was used. No evidence of asymmetry was detected in the data. 
Table 2. Analysis of the $V^{2}$ using geometrical models.

\begin{tabular}{|c|c|c|c|c|c|c|}
\hline \multirow[t]{2}{*}{ Instrument } & \multirow[t]{2}{*}{ Spectral band } & \multirow{2}{*}{$\begin{array}{c}t-t_{0} \\
\text { day }\end{array}$} & \multirow{2}{*}{$\begin{array}{c}\text { Single component model } \\
\text { UD diameter } \\
\text { [mas }]\end{array}$} & \multicolumn{3}{|c|}{ Double component model } \\
\hline & & & & $\begin{array}{c}\text { Unres. flux } \\
{[\%]}\end{array}$ & $\begin{array}{l}\text { UD flux } \\
{[\%]}\end{array}$ & $\begin{array}{l}\text { UD diam. } \\
\text { [mas] }\end{array}$ \\
\hline CHARA/CLASSIC & broad $K$ & 2.92 & $1 . \pm 0.2$ & - & - & - \\
\hline VLTI/PIONIER & broad $H$ & 12.81 & $0.6 \pm 0.1$ & - & - & - \\
\hline CHARA/CLASSIC & broad $K$ & 13.93 & $1.12 \pm 0.14$ & - & - & - \\
\hline VLTI/AMBER & $2.1 \pm 0.05 \mu \mathrm{m}$ & 28.76 & $2.58 \pm 0.3$ & $65 \pm 12$ & $35 \pm 8$ & $7.3 \pm 0.3$ \\
\hline VLTI/PIONIER & broad $H$ & 48.74 & $2.23 \pm 0.1$ & $83 \pm 9$ & $17 \pm 2$ & $8.5 \pm 0.2$ \\
\hline
\end{tabular}

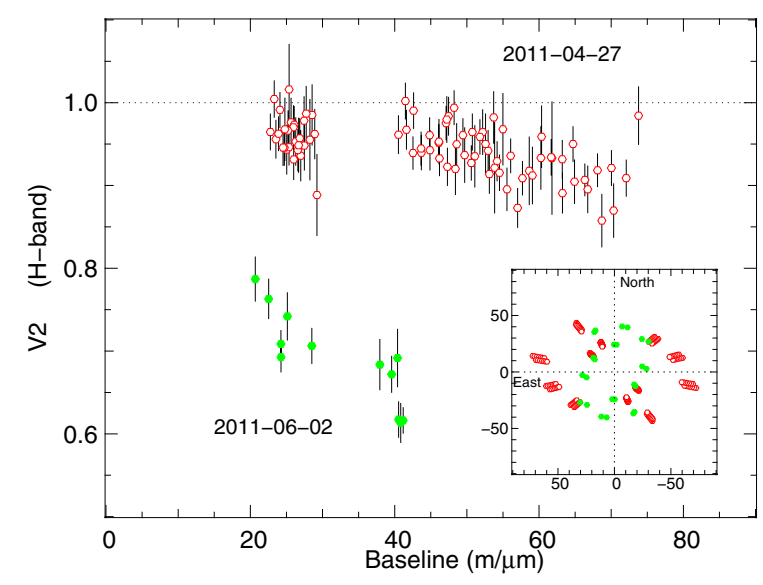

Fig. 3. $H$-band interferometric visibilities obtained with PIONIER at $t=12.81$ (open red) and $t=48.74$ (filled green). The corresponding $u v$-plane is displayed in the inset.

The first $K$-band CLASSIC measurement, obtained with $\mathrm{a} \sim 100 \mathrm{~m}$ baseline was consistent with a weakly resolved source, while the second set $(t=13.93 \mathrm{~d})$ with a baseline about twice as long shows a resolved source whose size does not seem to be dramatically changed. The first PIONIER measurements ( $t=12.81 \mathrm{~d}$ ) provided an $H$-band UD diameter that is significantly smaller than the CLASSIC $K$ band measurements, even taking the small time difference between the two measurements into account. This effect cannot be attributed to emission lines seen on these dates, which contribute less than $10-15 \%$ of the flux in the $H$ band and less than $5 \%$ in the $K$ band. A large-scale component with a rising flux contribution in the $K$ band may account for the observations, and is consistent with an $H-K$ flux difference of $0.33 \mathrm{mag}$ that is observed consistently during the event. Assuming a two-component model with a fixed contribution of $70 \pm 10 \%$ from a "stellar" source with a diameter of 0.6 mas, the extended $K$-band source should have a diameter larger than 1.5-2 mas, and therefore be almost fully resolved by CLASSIC. Assuming a distance of $D=3.5 \pm 1 \mathrm{kpc}$ from Schaefer et al. (2010), which may be a lower limit (Shore et al. 2011), the expansion velocity inferred from such an extended component is about $500 \mathrm{~km} \mathrm{~s}^{-1}$, while the $H$-band core expansion is estimated to be $\sim 100 \mathrm{~km} \mathrm{~s}^{-1}$ (Fig. 4). Interestingly, the $F W H M$ of the $\mathrm{Br} \gamma$ measured from the AMBER spectrum at $t=8.81 \mathrm{~d}$ is $590 \mathrm{~km} \mathrm{~s}^{-1}$, i.e. is consistent with our hypothesis of the expanding extended component (Fig. 5).

Then a second epoch follows during which AMBER interferometric data were obtained on two dates, yet they provide calibrated visibilities only at $t=28.76 \mathrm{~d}$. A single UD does not account well for the observed visibilities at $t=28.76 \mathrm{~d}$ (reduced $\left.\chi_{r}^{2}=35\right)$, the two-component model provides a better fit to the data $\left(\chi_{r}^{2}=5\right)$. This gives an upper limit for the expansion

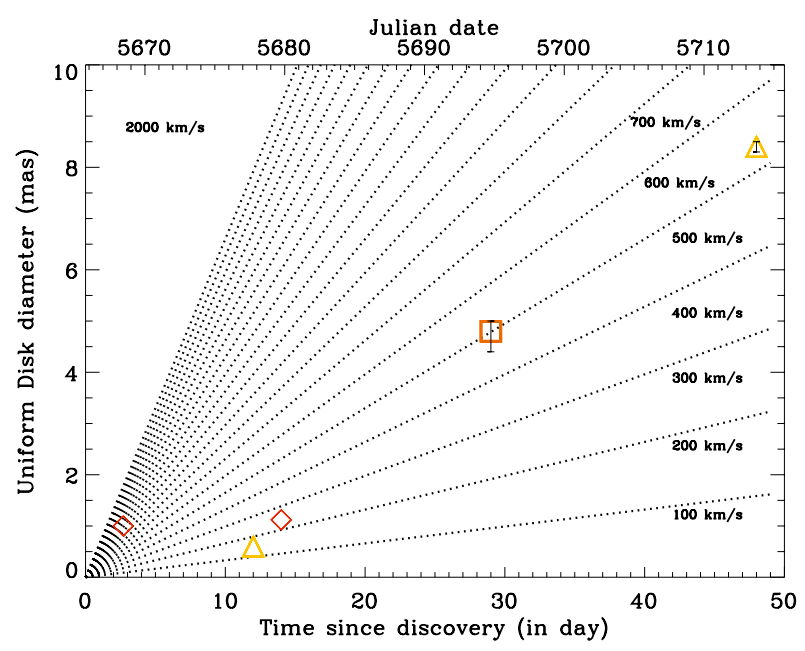

Fig. 4. Result of the uniform disk (UD) estimates from the continuum $V^{2}$ measurements from the various interferometers. Before $t=$ $20 \mathrm{~d}$, the source size is estimated using a single UD in the $H$ and $K$ bands (PIONIER: orange triangle; CLASSIC: red diamonds). The last points indicate the extended source in the double component model (AMBER: red square; PIONIER: orange triangle).

of the extended continuum component of $700 \mathrm{~km} \mathrm{~s}^{-1}$. It contrasts with the width of the $\mathrm{Br} \gamma$ line, for which we measured a $F W H M$ of $1050 \pm 50 \mathrm{~km} \mathrm{~s}^{-1}$. Furthermore, the Doppler velocity associated with the P-Cygni absorption in the line is found to be $-1450 \pm 100 \mathrm{~km} \mathrm{~s}^{-1}$ (Fig. 5). The $\mathrm{Br} \gamma$ line-forming region should therefore expand much faster, and a large visibility drop should be measured through the Br $\gamma$ line, as seen for RS Oph (Chesneau et al. 2007). However, the dispersed $\mathrm{Br} \gamma$ visibilities are only slightly lower than the nearby continuum, implying a moderate diameter increase of less than $10 \%$. One week later, the visibilities dropped in the line, indicating a large expansion of the $\mathrm{Br} \gamma$ line-forming region (taking the $25 \%$ increase in the line flux into account). At that time, the line $F W H M$ was measured to be $1600 \pm 50 \mathrm{~km} \mathrm{~s}^{-1}$, and the P-Cygni absorption indicated a wind velocity of $1800 \pm 100 \mathrm{~km} \mathrm{~s}^{-1}$. The differential phases show a complex structured signal that can be described by two opposite $\mathrm{S}$-shaped signals, with variations related to the baseline lengths and PA dependency. The pattern is symmetrical about the line center, with the width and amplitude of the signal increasing between the two dates, although the amplitude never went beyond $10^{\circ}$ (again by contrast with what was observed for RS Oph).

The last observations, which were performed at $t=48.74 \mathrm{~d}$ with PIONIER, bring complementary and crucial information, owing to the larger $u v$ coverage involved. The $H$ band source is now resolved well and departs from a simple model. A good fit $\left(\chi^{2}=1.1\right)$ is reached using the two-component model (Table 2). The extended component estimated expansion velocity in the 


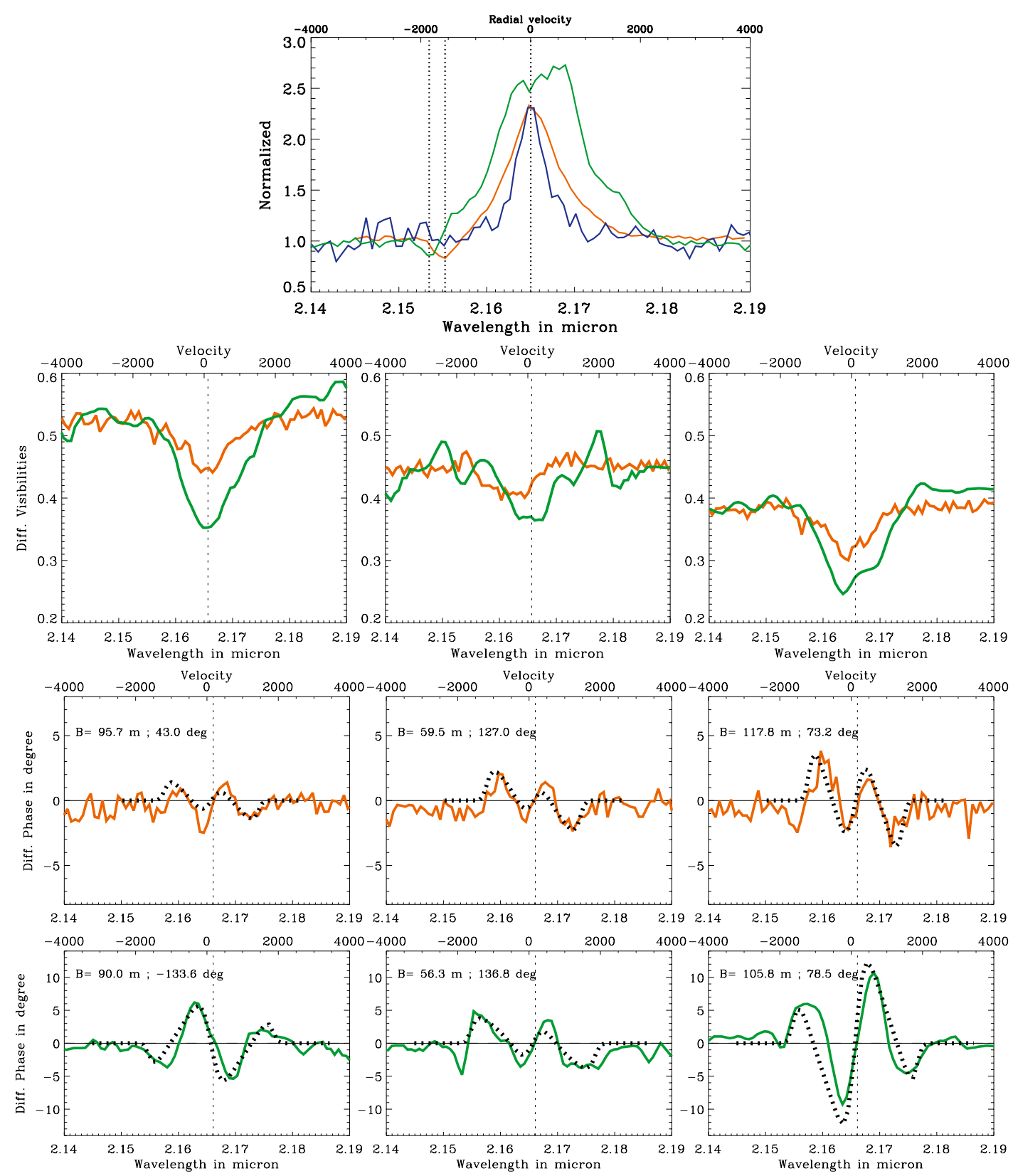

Fig. 5. AMBER data. Top: comparison between the $\operatorname{Br} \gamma$ line at $t=8.81$ (blue curve), $t=28.76 \mathrm{~d}$ (red curve), and $t=35.77 \mathrm{~d}$ (green curve). Bottom: above - differential visibility comparison between $t=28.76 \mathrm{~d}$ and $t=35.77 \mathrm{~d}$ (scaled to the continuum $V^{2}$ at $t=28.76 \mathrm{~d}$ ). Below: same with differential phases. The phases are compared with the phases from the model (dashed line, $\chi_{r}^{2}=1.1$ and 1.4 , compared to the red and green curves, respectively).

plane of the sky is lower than $700 \mathrm{~km} \mathrm{~s}^{-1}$. The closure phases do not exceed $2.5^{\circ}$. Moreover, using a flattened structure for the extended component does not improve the fit and constrains the aspect ratio to $1 \pm 0.07$. This implies that the complex yet weak phase signal seen by AMBER comes from a source with a predominantly symmetrical appearance.

\section{A face-on bipolar event}

The heterogeneous data set from different instruments provides intriguing data, and is unique in view of the complementary constraints provided for the analysis. The interpretation of these data can be divided into several key temporal steps.

- CLASSIC and PIONIER data obtained at $t=2.92 \mathrm{~d}, 12.81 \mathrm{~d}$ and $13.93 \mathrm{~d}$ show an extended $H$ and $K$ band source. That
T Pyx is resolved so early is intriguing. A hypothesis might be a light echo witnessing a close-by circumbinary environment, adding incoherent flux to the measurement. The expansion rate is then low between the first and second measurements, and a striking difference is observed between the $H$ and the $K$ band inferred diameters. This suggests an advanced decoupling between a shrinking optically thick core and an expanding free-free emitting optically thin envelope.

- The AMBER data obtained at $t=28.76 \mathrm{~d}$ provide evidence that the $\mathrm{Br} \gamma$ line-forming region projected onto the sky is formed close to the expanding continuum, but is more extended at $t=35.77 \mathrm{~d}$. The Doppler velocity accelerated from $1300 \mathrm{~km} \mathrm{~s}^{-1}$ to $1600 \mathrm{~km} \mathrm{~s}^{-1}$ in the time interval. A striking differential phase pattern is observed.

- The PIONIER dataset secured at $t=48.74 \mathrm{~d}$ is critical for establishing the two component nature of the emission, 
evidencing a large contribution from an unresolved stellar source and also an extended region whose appearance is circular and symmetric within error bars.

A face-on bipolar event could account for all of the information described above, in accordance with the determination of a low inclination for the system by Uthas et al. (2010). In particular, the differential phase pattern can be linked to the geometry and kinematics of the ejecta. We developed a "toy" model that provides a good match to the observations. Given two ad-hoc threedimensional distributions, one for the "emission" of the ejecta and one for the velocity field, we reconstructed intensity maps in narrow spectral bands in the emission line and then computed the corresponding visibilities and differential phases. The intensity map is created by considering that the matter was ejected during a brief outburst (best ascribed by a shell), propagating at $v_{r}(\theta)$. Consequently, the geometry is directly related to kinematics of the ejecta. We used the radial expansion law

$v_{r}(\theta)=v_{\text {pole }}+\left(v_{\text {eq }}-v_{\text {pole }}\right) \sin \theta$

where $\theta$ is the colatitude, and $v_{\text {pole }}$ and $v_{\text {eq }}$ are the polar and equatorial radial velocities.

We also considered an emission decreasing according to a power law of the distance. At a given epoch $t$, the 3D intensity distribution is proportional to

$I(r, \theta, \phi) \propto \frac{1}{r^{\alpha}} \exp \left[\frac{-\left(v_{r} t-r\right)^{2}}{2 \sigma_{r}^{2}}\right]$.

Using this model we were able to fit the $\operatorname{Br} \gamma$ differential visibilities and phases, as well as the line profile for the two epochs. The parameters of the best model are $i=15^{\circ}$, PA of the polar axis of $110^{\circ}, \alpha=2$, and the velocities for the two epochs:

$$
\begin{aligned}
& -v_{\text {pole }}=1200 \mathrm{~km} \mathrm{~s}^{-1} \text { and } v_{\text {eq }}=600 \mathrm{~km} \mathrm{~s}^{-1} \text { at } t=28.76 \mathrm{~d} ; \\
& -v_{\text {pole }}=1600 \mathrm{~km} \mathrm{~s}^{-1} \text { and } v_{\text {eq }}=700 \mathrm{~km} \mathrm{~s}^{-1} \text { at } t=35.77 \mathrm{~d} .
\end{aligned}
$$

The fit of the differential phases at the two epochs and the model images are presented in Fig. 5 and the model in Fig. 6. The polar and equatorial velocities are in good agreement with the Doppler and sky plane velocities estimated in Sect. 3. Furthermore, the PA of the equatorial plane overdensity is oriented in a direction similar to the PA of the faint X-ray nebula (Balman 2010).

The face-on bipolar nebula allows one to better understand the curious nebula scrutinized with HST (Schaefer et al. 2010; Shara et al. 1997, 1989). The knots are concentrated in a ring $\left(3.2-6^{\prime \prime}\right)$, that is expanding radially with a velocity in the restricted range of $500-700 \mathrm{~km} \mathrm{~s}^{-1}$ and with a mean radial velocity of about $500 \mathrm{~km} \mathrm{~s}^{-1}$ (O'Brien \& Cohen 1998). Deciphering between a projected sphere and a bipolar structure producing a dense, face-on ring is difficult, considering that radial velocity measurements of individual clumps are missing.

Some recent examples suggest that bipolarity in the ejecta of classical/recurrent novae may be relatively frequent: RS Oph (Ribeiro et al. 2009; Bode et al. 2007; Chesneau et al. 2007), V445 Pup (Woudt et al. 2009), V1280 Sco (Chesneau et al. 2008; Chesneau et al., in prep.) or HR Del (Harman \& O'Brien 2003). A significant difference does exist between the T Pyx and RS Oph environments: the lack of material around T Pyx, witnessed for instance by the lack of hard X-rays (Kuulkers et al. 2011), leads us to favor bipolarity induced by a process internal to the system, whether by the common envelope interaction with
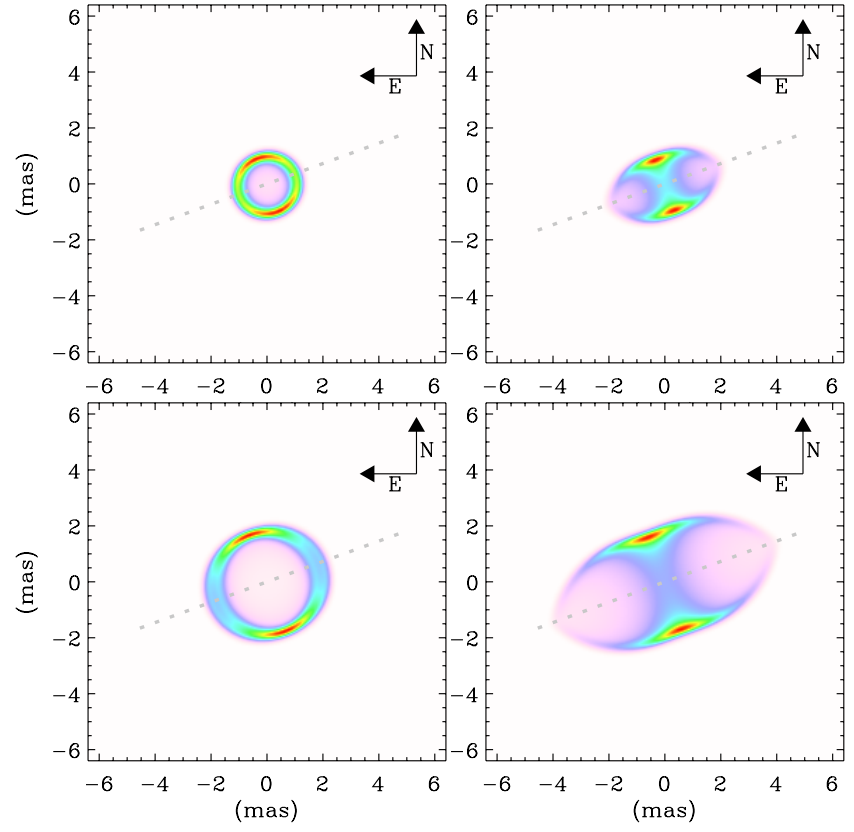

Fig. 6. $\mathrm{Br} \gamma$ bipolar-flow model (without central source) seen at $i=90^{\circ}$ (right) and $i=10^{\circ}$ (left, the best model) and PA $=110^{\circ}$ (best model).

the companion, since the development of the event is relatively slow, or by invoking an intrinsically bipolar ejection related to a spun-up central star (Porter et al. 1998; Lloyd et al. 1997).

Acknowledgements. The CHARA Array is funded by the National Science Foundation through NSF grant AST-0908253, by Georgia State University, the W. M. Keck Foundation, the Packard Foundation, and the NASA Exoplanet Science Institute. Research at the Physical Research Laboratory is funded by the Dept. of Space, Govt. of India. STR acknowledges partial support from NASA grant NNH09AK731.

\section{References}

Balman, Ş. 2010, MNRAS, 404, L26

Banerjee, D. P. K., \& Ashok, N. M. 2011, The Astronomer's Telegram, 3297, 1

Berger, J.-P., Zins, G., Lazareff, B., et al. 2010, SPIE Conf. Ser., 7734

Bode, M. F., Harman, D. J., O’Brien, T. J., et al. 2007, ApJ, 665, L63

Bonneau, D., Clausse, J.-M., Delfosse, X., et al. 2006, A\&A, 456, 789

Chesneau, O., Nardetto, N., Millour, F., et al. 2007, A\&A, 464, 119

Chesneau, O., Banerjee, D. P. K., Millour, F., et al. 2008, A\&A, 487, 223

Harman, D. J., \& O'Brien, T. J. 2003, MNRAS, 344, 1219

Kuulkers, E., Page, K. L., Ness, J.-U., et al. 2011, The Astronomer's Telegram, 3285,1

Le Bouquin, J.-B., Berger, J., Lazareff, B., et al. 2011, A\&A, in press DOI: $10.1051 / 0004-6361 / 201117586$

Lloyd, H. M., O'Brien, T. J., \& Bode, M. F. 1997, MNRAS, 284, 137

O’Brien, T. J., \& Cohen, J. G. 1998, ApJ, 498, L59

Petrov, R. G., Malbet, F., Weigelt, G., et al. 2007, A\&A, 464, 1

Porter, J. M., O’Brien, T. J., \& Bode, M. F. 1998, MNRAS, 296, 943

Ribeiro, V. A. R. M., Bode, M. F., Darnley, M. J., et al. 2009, ApJ, 703, 1955

Schaefer, B. E., Pagnotta, A., \& Shara, M. M. 2010, ApJ, 708, 381

Shara, M. M., Moffat, A. F. J., Williams, R. E., \& Cohen, J. G. 1989, ApJ, 337, 720

Shara, M. M., Zurek, D. R., Williams, R. E., et al. 1997, AJ, 114, 258

Shore, S. N., Augusteijn, T., Ederoclite, A., \& Uthas, H. 2011, A\&A, 533, L8

Tallon-Bosc, I., Tallon, M., Thiébaut, E., et al. 2008, SPIE Conf. Ser., 7013

ten Brummelaar, T. A., McAlister, H. A., Ridgway, S. T., et al. 2005, ApJ, 628, 453

Uthas, H., Knigge, C., \& Steeghs, D. 2010, MNRAS, 409, 237

Waagan, E., Linnolt, M., Bolzoni, S., et al. 2011, Central Bureau Electronic Telegrams, 2700, 1

Woudt, P. A., Steeghs, D., Karovska, M., et al. 2009, ApJ, 706, 738 